\title{
Non-Mosaic Trisomy 7 in Chorionic Villi and Trisomy 18 in the Fetus: An Extreme form of Mosaic Variegated Aneuploidy?
}

\author{
Annegret Geipel ${ }^{1}$, Thomas Eggermann ${ }^{2}$, Gisela Knöpfle ${ }^{3}$, Gesa Schwanitz $^{4}$, Ursula Pätzold ${ }^{4}$ and \\ Dagmar Hansmann ${ }^{5}$ \\ ${ }^{1}$ Department of Obstetrics and Prenatal Medicine, University Hospital Bonn, Germany \\ ${ }^{2}$ Institute of Human Genetics, University Hospital Aachen, Germany \\ ${ }^{3}$ Institute of Pathology, University Hospital Bonn, Germany \\ ${ }^{4}$ Institute of Human Genetics, University Hospital Bonn, Germany \\ ${ }^{5}$ Prenatal Medicine and Genetics, Meckenheim - Bonn, Germany
}

KEYWORDS Mosaic Variegated Aneuploidy. Trisomy 7. Trisomy 18. Parental Origin. Postzygotic Nondisjunction

\begin{abstract}
Mosaic variegated aneuploidy (MVA) is characterized by multiple trisomies. The parallel existence of two non-mosaic trisomies of two different chromosomes in the fetus and the placenta has not been published to date. We report here on a putative extreme form of MVA in a pregnancy with a non-mosaic trisomy 7 in CVS and a nonmosaic trisomy 18 in amniotic fluid. The trisomy 7 was not detected in amniocytes, but a non-mosaic trisomy 18 was diagnosed. Both aneuploidies were confirmed through STR typing of the respective tissue. We infer a postzygotic mitotic origin of both aneuplodies based on the observed reduction of maternal heterozygosity to homozygosity in the analysed markers. It is indeed well conceivable that mitotic errors occur in the early embryo briefly after differentiation into trophoblast and epiblast, resulting in a complete fetal-placental discordance such as the one observed in our case. However, the observation that trisomies 7 and 18 are among the most common aberrations in MVA would support our assumption that the complete discordance of the chromosomal complement in our case represents an extreme form of MVA.
\end{abstract}

\section{INTRODUCTION}

Nondisjunction of chromosomes or chromatids is a frequent failure of segregation and occurs at different steps of meiosis or during postzygotic mitoses. The further development of gametes and conceptuses depends on the chromosomes involved in nondisjunction and on the resulting monosomy or trisomy. A particular subgroup of aneuploidies are those resulting from sequential nondisjunction involving one chromosome and leading to tetrasomy or pentasomy. The cause may lie in either paternal or maternal nondisjunction or in malsegregation of two different uniparental chromosomes, thus causing double aneuploidy.

Mosaic variegated aneuploidy (MVA) refers to a condition in which multiple trisomies, rarely monosomies, occur within the same individual (Warburton et al. 1991). MVA has been reported

Correspondence should be addressed to:

Thomas Eggermann, Ph.D.

Institute of Human Genetics, RWTH Aachen

Pauwelsstr. 30 D-52074 Aachen, Germany

Phone: +49 2418088008

Fax: +49 2418082394

E-mail: teggermann@ukaachen.de in 29 patients (for review: Micale et al. 2000) and is thought to follow an autosomal recessive mode of inheritance in some cases. Its molecular pathogenesis is unclear, though some evidence indicates an association with premature centromere division (PCD) (Plage et al. 2001). Here we report a pregnancy with a non-mosaic trisomy 7 in CVS and a non-mosaic trisomy 18 in amniotic fluid and discuss our case in the context of MVA.

\section{CASE REPORT}

A 30-year-old woman, gravida 1, para 0 , underwent chorionic villous sampling (CVS) at $11+3$ weeks of gestation because of pathological ultrasound findings. There was no evidence of genetic or exogenous risk factors.

The ultrasound examination revealed an increased nuchal translucency of $10.2 \mathrm{~mm}$ (Fig. 1), hypoplasia of the heart's left ventricle, and a reverse flow in the ductus venosus. A second scan was performed at $12+2 \mathrm{WG}$, confirming the earlier pathological findings. In addition, generalized skin edema and a two vessel umbilical cord was diagnosed. The fetal biometry at both ultrasound scans is given in Table 1.

Initial cytogenetic investigations were 

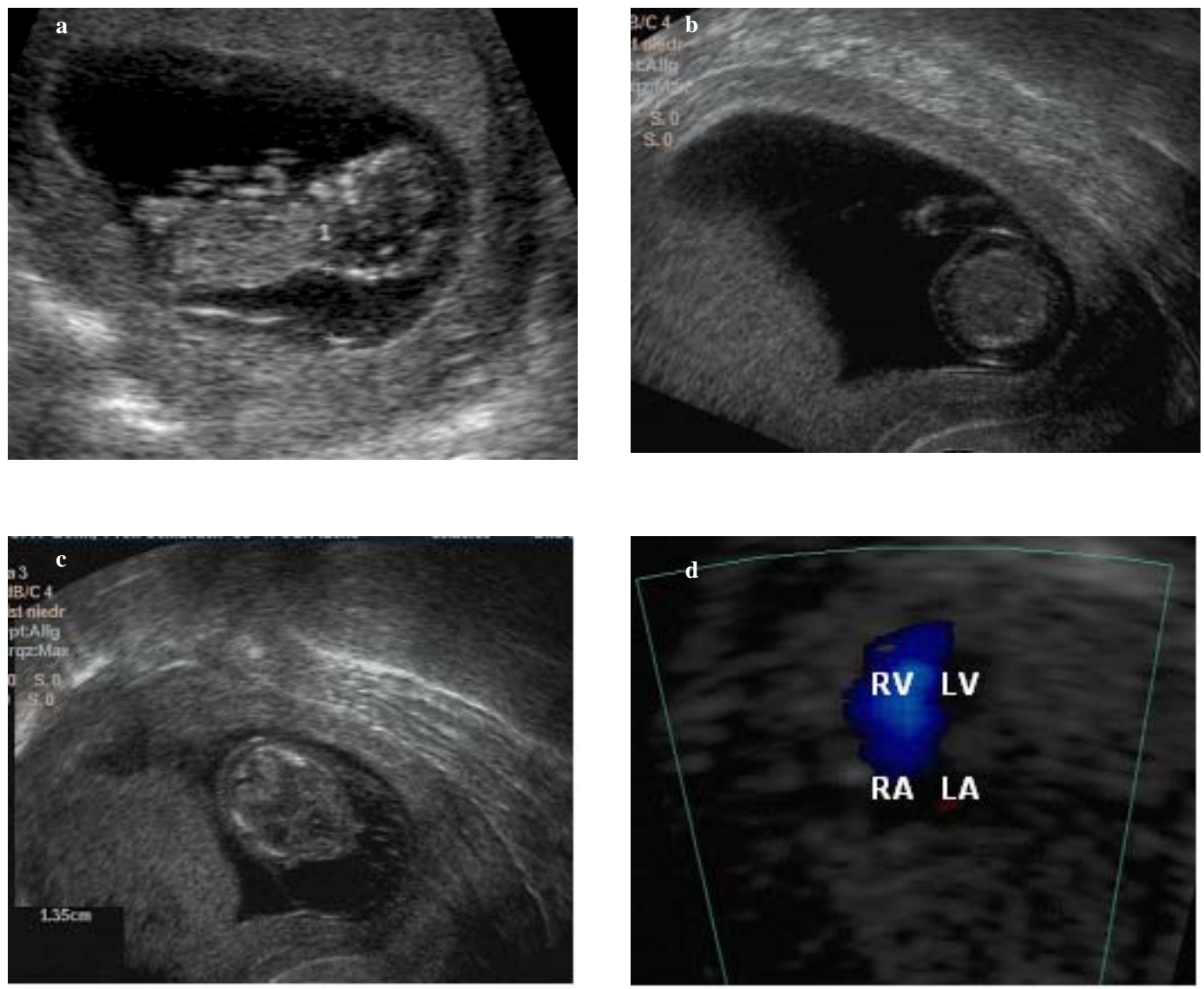

Fig. 1. a) Fetus at $11+3 \mathrm{WG}$; note the increased nuchal translucency $(\mathrm{NT}=10.2 \mathrm{~mm})$. b) Transverse section through the fetal abdomen (WG 12+2) demonstrating pronounced skin oedema. c) Transvaginal ultrasound shows an extreme nuchal translucency (WG 12+2). d) Four-chamber view of the heart (WG $12+2$ ). Flow only observed in the right ventricle.

Table 1: Developmental peculiarities of the fetus as documented by ultrasound. *fetal measurements compared with normal mean values and $5^{\text {th }} / 95^{\text {th }}$ percentile)

\begin{tabular}{|c|c|c|c|c|}
\hline \multirow[t]{2}{*}{ Biometry } & \multicolumn{2}{|c|}{$11+3 W G$} & \multicolumn{2}{|c|}{$12+2 W G$} \\
\hline & $m m$ & Percentile* $^{*}$ & $m m$ & Percentile* \\
\hline Crown-rump length (CRL) & 44.8 & $\vdash$ & 56.0 & \\
\hline Biparietal diameter (BPD) & 17.0 & $\mapsto \longmapsto$ & 20.0 & \\
\hline Frontooccipital diameter (FOD) & 22.0 & 1 & 26.0 & \\
\hline Head circumference (HC) & 64.4 & +1 & 72.3 & \\
\hline Femur length (FL) & 5.9 & 1 & 6.8 & \\
\hline Abdominal circumference (AC) & 50.5 & 1 & 49.2 & \\
\hline Nuchal translucency (NT) & 10.2 & +1 & 13.5 & $\theta$ \\
\hline Nasal bone (NB) & & & ible $(2$ & \\
\hline
\end{tabular}

(WG - weeks of gestation) 
performed in the extraembryonic tissue of the conceptus: in uncultured CVS cells, a non-mosaic trisomy $7(47, \mathrm{XX},+7)$ was detected in 11 metaphases by GTG banding. This observation was confirmed by interphase FISH in 30 nuclei. Analysis of 15 metaphases after long term culture ( 7 and 13 days) confirmed the trisomy 7 . There was no evidence for mosaicism or other aneuplodies (Table 2).

Based on the karyotype and the ultrasound findings, the parents decided on termination of pregnancy (TOP) after nondirective genetic counselling.

Amniotic fluid was drawn before TOP at $12+2$ WG to determine the fetal karyotype,. In contrast to the cytogenetic findings after CVS, trisomy 7 was not detected in amniocytes. However, after 10 days of cultivation spreads of GTG-banded chromosomes and FISH of interphase nuclei were analysed and non-mosaic trisomy 18 was diagnosed based on 6 metaphases (GTG) and 50 interphases, respectively (Table 2). Premature centromere division (PCD) was not observed in either CVS cells or amniocytes, although, due to the small numbers of analysed mitoses, it cannot be excluded.

Pathologic investigation after termination of pregnancy showed a generalized edema of the fetal skin, dolichocephalus, a hypoplastic left ventricle, a wrist drop in the right hand and a two vessel umbilical cord. The placenta was characterized by hydropic swelling of the villi, reduced vascularization, and calibre differences of the villous tree. The trophoblast consisted of 2 cell layers, which were particularly reduced in height.

Short tandem repeat (STR) typing was performed and confirmed each aneuploidy in the respective tissue (Table 3). In DNA isolated from a CVS culture, typing of chromosome 7 markers
Table 3: Results of STR typing in chorionic and amniotic DNA from our case: trisomy 7 in CVS and trisomy 18 in amniocytes. Maternal alleles duplicated in the fetus, thus corresponding to a trisomy, are printed in boldface. (n.a. not analysed)

\begin{tabular}{lrrrr}
\hline STR & Father & Mother & $\begin{array}{c}\text { chorionic } \\
\text { villi }\end{array}$ & $\begin{array}{c}\text { amnio- } \\
\text { cytes }\end{array}$ \\
\hline D7S493 & $193-212$ & $212-214$ & $193-214$ & $193-214$ \\
D7S483 & $165-174$ & $167-178$ & $167-174$ & $167-174$ \\
D7S460 & $174-182$ & 178 & $178-182$ & $178-182$ \\
D7S2251 & $146-164$ & $158-166$ & $164-166$ & $164-166$ \\
D7S636 & $132-149$ & $147-163$ & $147-149$ & $147-149$ \\
D7S2446 & $183-191$ & $199-204$ & $183-204$ & $183-204$ \\
D7S2452 & 192 & $192-200$ & 192 & 192 \\
D7S17B & 146 & 146 & 146 & 146 \\
D18S461 & $160-162$ & 162 & $160-162$ & $160-162$ \\
D18S70 & $230-240$ & $232-238$ & $232-240$ & $232-240$ \\
D18S390 & $160-166$ & $164-166$ & $160-164$ & $160-164$ \\
D18S535 & 127 & 130 & n.a. & $127-130$ \\
\hline
\end{tabular}

revealed two alleles with different intensities, supporting the cytogenetic diagnosis of trisomy 7. In amniotic fluid cells, analyses of chromosome 18 STRs also showed two alleles with different intensities, consistent with the cytogenetic diagnosis of trisomy 18 . In all informative markers the maternally inherited allele was markedly stronger than the paternal one. The occurrence of three different alleles, which would indicate a meiotic formation mechanism of the aneuploidies, was never observed. Instead, the reduction of maternal heterozygosity to homozygosity in all markers provides evidence for a postzygotic nondisjunctional error. There was no indication of cells trisomic for chromosome 7 in the amniotic fluid cells, nor was there any evidence of trisomy 18 cells in the CVS sample, either.

Typing of STR markers on chromosomes other than 7 and 18 confirmed that the DNA from both CVS and amniocyte samples originated from the same individual (data not shown).

Table 2: Frequency of trisomic cell lines in chorionic villus and amniocyte cell samples analysed by karyotyping and FISH methods.

*no additional results for chromosome 18; **decreased hybridisation efficency.

\begin{tabular}{|c|c|c|c|c|c|c|c|c|c|}
\hline \multicolumn{10}{|c|}{ Investigated cell system } \\
\hline & \multicolumn{3}{|c|}{$C V S(W G 11+3)$} & \multicolumn{4}{|c|}{$A C(W G 12+2)$} & & \\
\hline & $\begin{array}{c}\text { Karyotype } \\
\text { analysis } \\
47, X X,+7\end{array}$ & $n$ & $\begin{array}{c}\text { Interphasel } \\
\text { Metaphase FISH } \\
\text { Trisomy } 7 * \\
\end{array}$ & $n$ & $\begin{array}{l}\text { Karyotype } \\
\text { analysis } \\
47, X X,+18\end{array}$ & $n$ & $\begin{array}{c}\text { Interphasel } \\
\text { Metaphase FISH } \\
\text { Trisomy } 7 \text { and } 18\end{array}$ & \multicolumn{2}{|r|}{$n$} \\
\hline $\begin{array}{l}\text { Direct } \\
\text { preparation }\end{array}$ & $100 \%$ & 11 & $86 \%$ & $35 * *$ & - & - & - & - & - \\
\hline $\begin{array}{l}\text { Long term } \\
\text { cell culture }\end{array}$ & $100 \%$ & 15 & - & - & $100 \%$ & 38 & $\begin{array}{r}\text { Trisomy } 7 \\
\text { Trisomy } 18\end{array}$ & $\begin{array}{r}0 \% \\
91 \%\end{array}$ & $\begin{array}{r}132 \\
56\end{array}$ \\
\hline
\end{tabular}




\section{DISCUSSION}

We were only partially able to analyse the phenotype of our patient due to the early termination of the pregnancy and to the mode of termination. Clinical features such as early developmental retardation, heart malformation and skin edema have been diagnosed in a number of autosomal trisomies. However, some features of the fetus in this case are more specific and have frequently been observed in fetal cases of trisomy 18 (Schinzel 2001), namely dolichocephalus, wrist drop, and the reduced number of umbilical cord blood vessels. The pathologic changes of the chorionic villi are typical for a number of autosomal trisomies (Lukas et al. 1989).

The postzygotic origin of the trisomy 7 cell line in our case is in accordance with published findings: Studies on trisomy 7 show that it frequently originates from mitotic errors (for review: (Mergenthaler et al. 2000). Furthermore, trisomy 7 has repeatedly been reported to be involved in CVS mosaicism (for review: Gardner and Sutherland 2004). In these cases, the percentage of trisomic cells ranged from 7 to $100 \%$ while the fetus itself carried a normal chromosomal complement and was judged inconspicuous, except for those cases with uniparental disomy 7 (Mergenthaler et al. 2000).

In contrast, a postzygotic mitotic nondisjunction has been delineated in less than $8 \%$ of cases for trisomy 18 (Bugge et al. 1998); the majority of trisomies 18 originates from a maternal meiosis II error.

In our case we cannot exclude that both trisomies developed postzygotically simply by chance. This would, however, be an extremely rare event. Instead, we assume that our case represents the extreme form of MVA. As mentioned previously, all MVA patients reported thus far have shown a somatic mosaicism for different chromosomes, pointing to postzygotic nondisjunction in a later stage of embryonic development. However, it is well conceivable that mitotic errors in the early embryo shortly after differentiation into trophoblast and epiblast might occur, resulting in a complete fetal-placental discordance as observed in our case. The observation that trisomies 7 and 18 are among the most common aberrations in MVA patients further supports our conclusion that the complete discordance of the chromosomal complement in our case represents the extreme end of MVA.

\section{REFERENCES}

Bugge M, Collins A, Petersen MB, Fisher J, Brandt C, Hertz JM, Tranebjaerg L, de Lozier-Blanchet C, Nicolaides P, Brondum-Nielsen K, Morton N, Mikkelsen M 1998. Non-disjunction of trisomy 18. Hum Mol Genet, 7: 661-669.

Gardner RJM, Sutherland GR 2004 Chromosome Abnormalities and Genetic Counselling. $3^{\text {rd }}$ Edition, Oxford: Oxford University Press.

Lukas U, Gamerdinger F, Schwanitz G 1989. Growth retardation of the fetus combined with developmental abnormalities in the extraembryonic tissue in correlation with different chromosome abnormalities. Auxologie, 88: 387-393.

Mergenthaler S, Wollmann HA, Burger B, Eggermann K, Kaiser P, Ranke MB, Schwanitz G, Eggermann T 2000. Formation of uniparental disomy 7 delineated from new cases and a UPD7 case after trisomy 7 rescue. Ann Génét, 43: 15-21.

Micale MA, Schran D, Emch S, Kurczynski TW, Rahman N, van Dyke DL 2007. Mosaic variegated aneuploidy without microcephaly: implications for cytogenetic diagnosis. Am J Med Genet, 143A: 1890-1893.

Plaja A, Vendrell T, Smeets D, Sarret E, Gili T, Catala V, Mediano C, Scheres JM 2001. Variegated aneuploidy related to premature centromere division (PCD) is expressed in vivo and is a cancer-prone disease. Am J Med Genet, 98: 216-223.

Schinzel A 2001. Cataloque of Unbalanced Chromosome Aberrations in Man. $2^{\text {nd }}$ Edition, Berlin, New York: de Gruyter.

Warburton D, Anyane-Yeboa K, Takerka P, Yu CY, Olsen D 1991. Mosaic variegated aneuploidy with microcephaly: a new human mitotic mutant. Ann Génét, 34: 287-292. 